\title{
SEARCHING FOR YOUNG JUPITER ANALOGS AROUND AP COL: L-BAND HIGH-CONTRAST IMAGING OF THE CLOSEST PRE-MAIN-SEQUENCE STAR*
}

\author{
Sascha P. Quanz ${ }^{1}$, Justin R. Crepp ${ }^{2}$, Markus Janson ${ }^{3}$, Henning Avenhaus ${ }^{1}$, \\ Michael R. MEYeR ${ }^{1}$, AND LynNe A. Hillendrand ${ }^{2}$ \\ ${ }^{1}$ Institute for Astronomy, ETH Zurich, Wolfgang-Pauli-Strasse 27, 8093 Zurich, Switzerland; quanz@astro.phys.ethz.ch \\ 2 Department of Astrophysics, California Institute of Technology, 1200 East California Boulevard, Pasadena, CA 91125, USA \\ ${ }^{3}$ Department of Astrophysical Sciences, Princeton University, Princeton, NJ 08544, USA \\ Received 2012 March 20; accepted 2012 May 30; published 2012 July 17
}

\begin{abstract}
The nearby M-dwarf AP Col was recently identified by Riedel et al. as a pre-main-sequence star (age 12-50 Myr) situated only $8.4 \mathrm{pc}$ from the Sun. The combination of its youth, distance, and intrinsically low luminosity make it an ideal target to search for extrasolar planets using direct imaging. We report deep adaptive optics observations of AP Col taken with VLT/NACO and Keck/NIRC2 in the $L$ band. Using aggressive speckle suppression and background subtraction techniques, we are able to rule out companions with mass $m \geqslant 0.5-1 M_{\text {Jup }}$ for projected separations $a>4.5 \mathrm{AU}$, and $m \geqslant 2 M_{\text {Jup }}$ for projected separations as small as $3 \mathrm{AU}$, assuming an age of $40 \mathrm{Myr}$ using the COND theoretical evolutionary models. Using a different set of models, the mass limits increase by a factor of $\gtrsim 2$. The observations presented here are the deepest mass-sensitivity limits yet achieved within 20 AU on a star with direct imaging. While Doppler radial velocity surveys have shown that Jovian bodies with close-in orbits are rare around M-dwarfs, gravitational microlensing studies predict that $17_{-9}^{+6} \%$ of these stars host massive planets with orbital separations of 1-10 AU. Sensitive high-contrast imaging observations, like those presented here, will help to validate results from complementary detection techniques by determining the frequency of gas giant planets on wide orbits around M-dwarfs.
\end{abstract}

Key words: stars: formation - planets and satellites: formation - planets and satellites: detection - stars: individual (AP Col)

Online-only material: color figures

\section{INTRODUCTION}

The high-contrast imaging technique has provided some remarkable extrasolar planet discoveries over the past few years (e.g., Chauvin et al. 2005; Marois et al. 2008, 2010; Lagrange et al. 2010). Direct detection allows us not only to infer orbital separations and eventually determine orbits (Crepp et al. 2011), but also to study extrasolar planets in detail through multi-color photometry and spectroscopy (Mohanty et al. 2007; Quanz et al. 2010; Hinz et al. 2010; Janson et al. 2010; Bowler et al. 2010). Investigation of the small sample of directly imaged planets known to date has already revealed unexpected results, including the suggested presence of thick cloud layers (Skemer et al. 2011), enhanced metallicity, and indications of non-equilibrium chemistry (Barman et al. 2011). Such observations present a challenge to theoretical atmospheric models (Skemer et al. 2012).

Dedicated imaging surveys have now searched host stars of all spectral types, with an initial emphasis on FGK stars (Masciadri et al. 2005; Biller et al. 2007; Kasper et al. 2007; Lafrenière et al. 2007a; Chauvin et al. 2010; Heinze et al. 2010). The fact that the first handful of directly imaged planets have been found orbiting A-stars provides an unambiguous clue that the efficiency of the planet formation process depends upon host star mass. Indeed, extrapolation of the Doppler radial velocity planet population to separations accessible to high-contrast imaging instruments predicts that A-type stars are ideal targets, even though they are intrinsically bright (Crepp et al. 2012). Massive stars have a high

\footnotetext{
* Based on observations collected at the European Organisation for Astronomical Research in the Southern Hemisphere, Chile, under program number 288.C-5005(A).
}

gas giant planet occurrence rate, and a propensity to form more massive planets with long orbital periods (Johnson et al. 2007). While the same line of reasoning suggests a relative paucity of giant planets orbiting M-stars (Kennedy \& Kenyon 2008), initial results from gravitational microlensing surveys suggest a significantly higher frequency of planets located beyond the snow-line (Gould et al. 2010; Cassan et al. 2012) compared to rates of close-in planets inferred from Doppler measurements (Johnson et al. 2010; Bonfils et al. 2011).

High-contrast imaging observations will soon be able to rectify any discrepancies found by indirect planet detection techniques. Planned instruments and new adaptive optics (AO) systems at the Very Large Telescope (VLT; Beuzit et al. 2006), Gemini South (Macintosh et al. 2007), Palomar (Hinkley et al. 2011), the Large Binocular Telescope (LBT; Esposito et al. 2012), and Subaru (Tamura 2009) will generate unprecedented contrast levels, providing direct access to young planets with mass $m \approx 0.5 M_{\text {Jup }}$. It is imperative to reach this level of sensitivity because the "bottom-to-top" formation paradigm of core accretion (Pollack et al. 1996) predicts an overall planet occurrence rate that rises steadily with decreasing planet mass. In other words, access to sub-Jovian-mass bodies significantly enhances the prospects for detecting an extrasolar planet around any type of star.

Youth and distance are important parameters to consider when prioritizing high-contrast imaging targets. With an accurately measured distance of only $8.39 \pm 0.07 \mathrm{pc}$ and estimated age between 12 and $50 \mathrm{Myr}$, the nearby M4.5 flare star AP Col (R.A.: $06^{\mathrm{h}} 04^{\mathrm{m}} 52^{\mathrm{s}} .16$; decl.: $-34^{\circ} 33^{\prime} 36^{\prime \prime} 0$ (J2000, E2000)) has recently been identified as the closest pre-main-sequence star to the Sun (Riedel et al. 2011). Based on its apparent age and 
space motion, AP Col is most likely a member of the $\sim 40 \mathrm{Myr}$ old Argus/IC 2391 Association (Riedel et al. 2011). Stellar companions have been ruled out by lucky imaging observations prior to establishing its proximity and youth (Bergfors et al. 2010). Given its (extremely) convenient properties, AP Col is an ideal target to search for gas giant planets by means of direct imaging.

In this paper, we present the results from two different sets of deep high-contrast imaging observations taken at the VLT and Keck. The observations were carried out in the $L$ band which, compared to shorter near-infrared (NIR) wavelengths, provides a higher Strehl ratio and more favorable star-planet flux ratio, while also maintaining manageable thermal background levels from the sky and instrument optics. Despite generating the deepest direct imaging mass sensitivity yet achieved for orbital separations on the scale of the solar system $(a>3 \mathrm{AU})$, we find no evidence for the existence of gas giant planets. We discuss the implications of these observations to the extent that they support the notion that Jovian bodies appear to be rare around M-dwarfs.

\section{OBSERVATIONS AND DATA REDUCTION}

\subsection{VLT/NACO}

Imaging data were acquired on UT 2011 December 4, with the AO-fed, high spatial resolution camera NACO mounted on ESO's VLT/UT4 8.2 m telescope at Paranal (Lenzen et al. 2003; Rousset et al. 2003). All images were unocculted and taken with the L27 camera (plate scale $\sim 27.15$ mas pixel $^{-1}$ ) using the $L^{\prime}$ filter $\left(\lambda_{c}=3.8 \mu \mathrm{m}, \Delta \lambda=0.62 \mu \mathrm{m}\right)$. We used pupil stabilization mode to enable angular differential imaging (ADI; Marois et al. 2006).

To enhance the signal-to-noise ratio $(\mathrm{S} / \mathrm{N})$ of potential companions, we chose to moderately saturate the stellar point spread function (PSF) core. Unsaturated images were also acquired to calibrate the photometry. Detector reads were recorded individually ("cube mode"). The integration time was set to $0.2 \mathrm{~s}$ per read (unsaturated reads had an exposure time of $0.1 \mathrm{~s}$ ). We obtained 134 raw data cubes for AP Col in total. Each cube consisted of 64 reads. Between cubes we moved the telescope by $\approx 9^{\prime \prime}$ on the sky, executing a five-point dither pattern to facilitate removal of background noise from the sky and instrument optics. Individual detector reads were checked for open loops and poor AO correction. We discarded one complete cube as well as several individual reads from other cubes. The remaining reads were median combined in stacks of 32, yielding 265 science images with an effective integration time of $32 \times$ $0.2 \mathrm{~s}$ each. We measure the PSF FWHM in unsaturated images to be $\sim 4.3$ pixels $\left(\approx 0^{\prime} .12\right)$, which is comparable to the theoretical diffraction limit, $\Theta \approx \lambda_{c} / D \approx 0^{\prime \prime} 10$.

Our basic data reduction steps (bad pixel correction, sky subtraction) are described in Quanz et al. (2010, 2011). We use the LOCI algorithm to subtract the stellar PSF (Lafrenière et al. 2007b). Following the same naming convention as in the original paper, we set pertinent reduction parameters to the following values: $\mathrm{FWHM}=4.5$ pixels, $N_{\delta}=0.75, d r=5, N_{A}=300$.

\subsection{Keck/NIRC2}

We also observed AP Col from Keck Observatory on UT 2012 January 7. Using the $10 \mathrm{~m}$ Keck II telescope and AO system (Wizinowich et al. 2004), we acquired images with the Near InfraRed Camera (NIRC2; PI: Keith Matthews) in the $L^{\prime}$ filter $\left(\lambda_{c}=3.8 \mu \mathrm{m}, \Delta \lambda=0.7 \mu \mathrm{m}\right)$. A total of 67 images were
Table 1

Summary of Deep $L^{\prime}$ Imaging Observations of AP Col

\begin{tabular}{|c|c|c|}
\hline Parameter & VLT/NACO & Keck/NIRC2 \\
\hline No. of detector reads $\times$ exp. time & $64 \times 0.2 \mathrm{~s}$ & $300 \times 0.106 \mathrm{~s}$ \\
\hline No. of data cubes/stacked images & 134 cubes & 67 images \\
\hline Parallactic angle start/end & $-50.11 /+59.70$ & $-16.24 /+5.59$ \\
\hline Airmass range & $1.02-1.05$ & $1.72-1.78$ \\
\hline Typical DIMM seeing & $0 ! 7 \ldots 1^{\prime \prime} .0$ & $\sim 0{ }^{\prime} 6$ \\
\hline PSF FWHM $^{\mathrm{a}}$ & $\sim 0^{\prime \prime} 12\left(0^{\prime \prime} .10\right)$ & $\sim 0.09(0 . .08)$ \\
\hline$\langle E C\rangle_{\text {mean }}^{\mathrm{b}}$ & $19.1 \%$ & \\
\hline
\end{tabular}

Notes.

${ }^{a}$ Measured FWHM and theoretical diffraction-limited FWHM in parenthesis.

b Average value of the coherent energy of the NACO/PSF in data cubes. Calculated by the Real Time Computer of the AO system.

obtained, each consisting of 300 reads with $0.106 \mathrm{~s}$ integration time per read. All images were in the linear detector regime and recorded with the narrow camera setting which provides a plate scale of 9.963 mas pixel $^{-1}$ (Ghez et al. 2008). Like the VLT data, we did not use a coronagraph. Vertical angle mode was used to allow for ADI operation. The AO system was running at a refresh rate of $438 \mathrm{~Hz}$. The FWHM of the PSF was 9.1 pixels $\left(\approx 00^{\prime} 09\right)$, i.e., close to the theoretical diffraction limit of $\Theta \approx 0$.' 08 .

Raw images were processed using standard techniques to remove dark current, flat field, replace hot-pixel values, and align the target star. In the same manner as described in the previous section with the VLT data, we used the LOCI algorithm on the 67 images to subtract the stellar PSF. The only difference was the value for the FWHM which was set to 9 pixels for the Keck data. Table 1 shows a summary of relevant observing parameters for both the VLT and the Keck data sets.

\section{RESULTS}

We find no evidence for point sources in either the VLT or Keck data sets, in raw or processed images. Our checks for real companions did initially show potential candidates in the VLT data. However, they subsequently disappeared when combining only a fraction of the final images, and other bright speckles showed up instead.

\subsection{Derivation of Contrast Curve}

A comparison of the $5 \sigma$ contrast levels obtained with NACO and NIRC2 is shown in Figure 1. Contrast is defined as

$$
\Delta L^{\prime}(r)=2.5 \cdot \log _{10} \frac{F_{*}}{5 \sigma(r) / \sqrt{\pi r_{\text {ap }}^{2}}},
$$

where $F_{*}$ is the mean stellar flux per pixel in an aperture with radius $r_{\mathrm{ap}}=3$ pixels (VLT/NACO) or $r_{\mathrm{ap}}=5$ pixels (Keck/NIRC2), and $\sigma$ is the standard deviation of the pixels within centro-symmetric annuli centered on the star, which are twice as wide as the aperture radius. The VLT/NACO observations provide slightly deeper contrast compared to Keck as a result of lower background levels, more field rotation, and lower airmass. We focus on these data for the remainder of the analysis.

To derive our final detection limits, we insert fake planets of known brightness into individual NACO frames. Two planets of the same brightness are inserted at the same radial distance $\left(0.3,0^{\prime} .4,0^{\prime} .5,0^{\prime} .6,0^{\prime} .8,1^{\prime \prime} .0,1^{\prime \prime} .5\right.$, and $\left.2^{\prime \prime} .0\right)$, but at opposing 


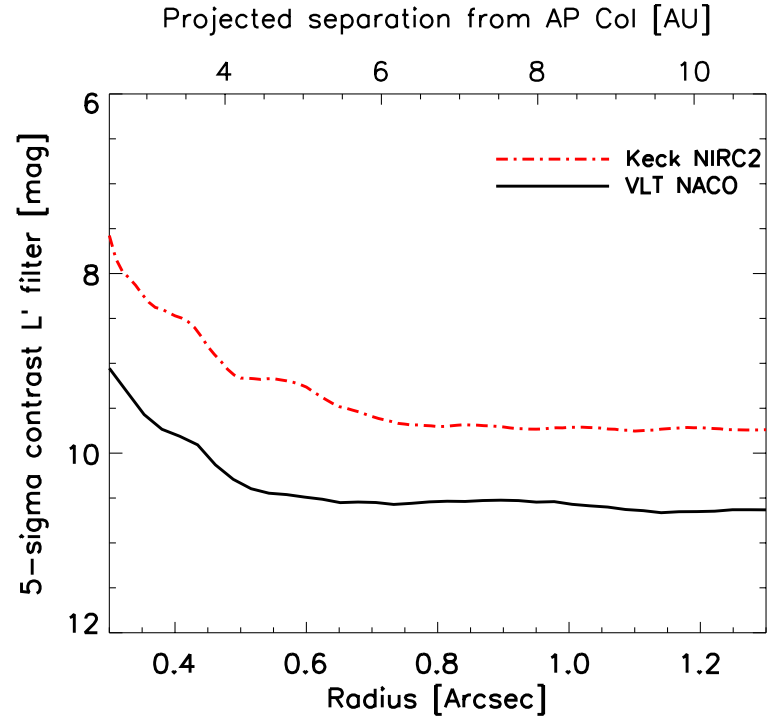

Figure 1. Comparison between the $5 \sigma$ contrast curves obtained with Keck/NIRC2 and VLT/NACO.

(A color version of this figure is available in the online journal.)

sides from the central star. The $\mathrm{S} / \mathrm{N}$ of the planets in the final LOCI-processed image is computed via

$$
\left(\frac{\mathrm{S}}{\mathrm{N}}\right)_{\text {Planet }}=\frac{F_{\text {Planet }}}{\sigma / \sqrt{\pi r_{\mathrm{ap}}^{2}}},
$$

where $F_{\text {Planet }}$ is the measured planet flux in an aperture with radius $r_{\mathrm{ap}}=3$ pixels and $\sigma$ is the standard deviation of the pixels within a 7 pixel wide arc between the two fake planets and centered at the same radial distance. By inserting two planets simultaneously and having two arcs for the noise estimate between the planets, we obtain four different values for $(\mathrm{S} / \mathrm{N})_{\text {Planet. }}$. To be conservative, we chose the combination yielding the lowest value as our reference. For a given radial separation we lowered the brightness of the planets in steps of $0.5 \mathrm{mag}$ until $(\mathrm{S} / \mathrm{N})_{\text {Planet }}<5$. The signal to noise of the next brightest planet was then used for calibration and the values extrapolated to a level that corresponds to $(\mathrm{S} / \mathrm{N})_{\text {Planet }}=5$. These values are shown in Figure 2.

We emphasize that injecting fake planets of various brightness and retrieving them is the only way to obtain an accurate estimate for the achieved detection limit. LOCI removes flux from potential companions (Lafrenière et al. 2007b) as a function of separation from the star, LOCI input parameters, and brightness of the companions themselves (Pueyo et al. 2012). There are no analytical methods for predicting and correcting for flux losses.

The limiting magnitude in the VLT/NACO data approaches $L_{\text {limit }}^{\prime} \approx 16.7 \mathrm{mag}$ at a separation of $\approx 0 ! 7$, beyond which the sensitivity becomes flat indicating that we have reached the floor set by thermal background noise (Figure 2). Sensitivity is dominated by subtraction residuals closer to the star where the detection limits are governed more so by residual scattered light.

\subsection{Detection Limits using COND Models}

To convert our on-sky contrast into mass sensitivity, we must first estimate the $L^{\prime}$ magnitude of AP Col. Using color transformations provided by the 2MASS team, ${ }^{4}$ we estimate AP Col's $K$ magnitude to be $K=6.88 \pm 0.05$ mag, based on the apparent $K_{s}$ value of $K_{s}=6.87 \pm 0.02$ mag (Cutri et al. 2003). A typical M4.5 star has an approximate $K-L^{\prime}$ color of $0.4 \mathrm{mag}$ (Cox 2000). Thus, AP Col has an $L^{\prime}$ magnitude of $L^{\prime} \approx 6.48 \pm 0.05 \mathrm{mag}$.

Figure 2 (left-hand panel) compares our detection limits with the predicted $L^{\prime}$ magnitudes for $40 \mathrm{Myr}$ objects having masses of $0.5,1$, and $2 M_{\text {Jup }}$ based on the COND models (Baraffe et al. 2003). We select this specific age because Riedel et al. (2011) derive a most likely age between 12 and $50 \mathrm{Myr}$ for AP Col, and membership with the $\sim 40$ Myr old Argus/IC 2391 Association seems plausible from AP Col's space motion. Our data are sufficiently sensitive to detect objects with masses between 0.5 and $1 M_{\text {Jup }}$ (or greater) for separations $\geqslant 4.5 \mathrm{AU}$. Inward of 4.5 $\mathrm{AU}$, as close as $\sim 3 \mathrm{AU}$, objects with masses between 1

\footnotetext{
4 http://www.astro.caltech.edu/ jmc/2mass/v3/transformations/
}
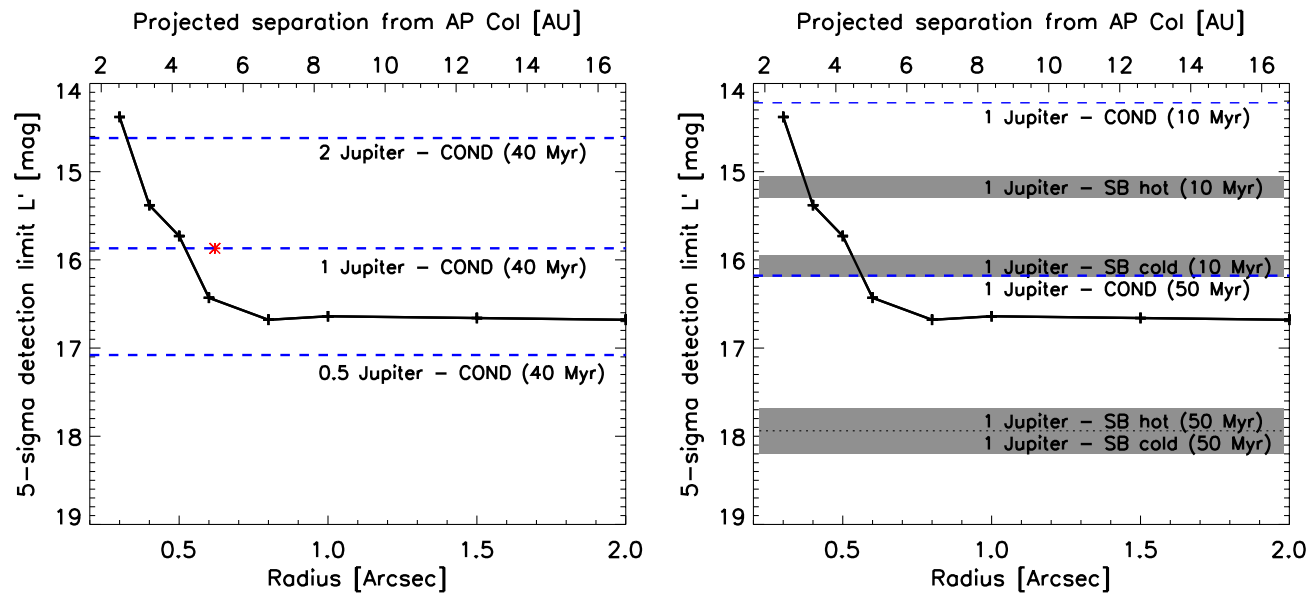

Figure 2. $5 \sigma$ detection limit in $L^{\prime}$ magnitudes for our VLT/NACO data as a function of separation from AP Col (black crosses and curve). Left: overplotted as horizontal dashed lines are predicted $L^{\prime}$ magnitudes for $40 \mathrm{Myr}$ old objects with masses of $0.5,1$, and $2 M_{\text {Jup }}$ at the distance of AP Col based on the COND models (Baraffe et al. 2003). The red asterisk shows the location of a young Jupiter analog (i.e., $1 M_{\mathrm{Jup}}$ at 5.2 AU projected separation). Right: overplotted are predictions from theoretical models for a $1 M_{\text {Jup }}$ object with an age of 10 or $50 \mathrm{Myr}$. The blue dashed lines are based on the COND models. The gray shaded areas are predictions from the hot and cold start models from Spiegel \& Burrows (2012), where the spread in magnitude for a given model, i.e., the width of the gray shaded areas, shows the difference between atmospheres with and without hybrid clouds. All models assume solar metallicity.

(A color version of this figure is available in the online journal.) 


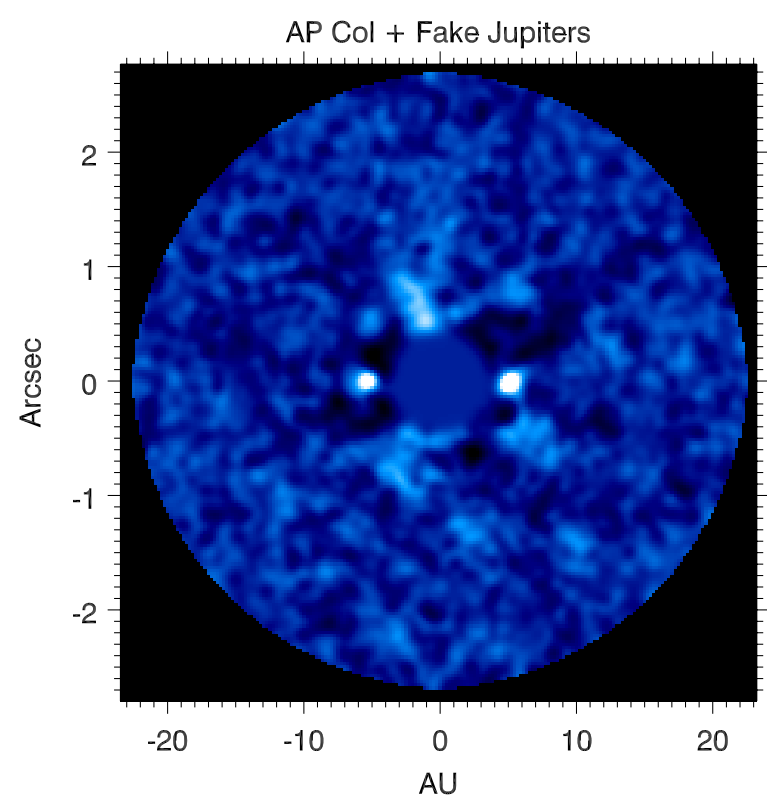

Figure 3. Final VLT/NACO image after a fake "Jupiter" has been inserted in the raw images at two different positions with a projected separation of $5.2 \mathrm{AU}$ from the central star. The brightness of the "planets" corresponds to a $1 M_{\text {Jup }}$ object with an age of $40 \mathrm{Myr}$ based on the COND models. Both "planets" are clearly detected with a signal to noise of $\sim 9.6$ and $\sim 12.1$, respectively. The innermost regions that are dominated by subtraction residuals have been masked out.

(A color version of this figure is available in the online journal.)

and $2 M_{\text {Jup }}$ (or greater) are detectable. Based on these model predictions, this is the first time that a young Jupiter analog could have been detected by means of direct imaging around another star. This is also demonstrated in Figure 3, where we have inserted two fake planets in the raw images at a projected separation of 5.2 AU and re-run the data reduction. The planets' brightness corresponds to a $1 M_{\text {Jup }}$ object with an age of $40 \mathrm{Myr}$ based on the COND models. It clearly shows that we would have detected such an object in our data.

To assess the likelihood of imaging a planet during a single epoch observation, the following relation can be used:

$$
P_{\text {detect }}(a)=\cos (\arcsin (\mathrm{IWA} / a)),
$$

where $P_{\text {detect }}$ is the detection probability, $a$ the semimajor axis of the planet in AU, and IWA is the inner working angle (in AU) where the contrast curves allows for the detection of this planet. This relation is only valid for circular orbits and assumes uniform sensitivity in azimuth, but takes into account all possible orbital inclinations. For eccentric orbits $P_{\text {detect }}$ would be higher for any given $a$, as the planet would spend more time close to apastron where it is easier to detect.

In Figure 4, we plot the detection probability for a $1 M_{\mathrm{Jup}}$ planet and a $2 M_{\text {Jup }}$ planet using IWA $=4.5 \mathrm{AU}$ for the $1 M_{\mathrm{Jup}}$ case and IWA $=3 \mathrm{AU}$ for the $2 M_{\text {Jup }}$ case (see, left-hand plot in Figure 2). It is clear that we had a $\sim 50 \%$ chance to detect (at greater than $5 \sigma$ ) a young Jupiter analog at 5.2 AU, assuming one is present. The probability rises quickly for more massive or more distant planets.

\subsection{Comparison of Theoretical Models}

In the right-hand plot of Figure 2, we have overplotted in horizontal lines and shaded regions the magnitudes for a $1 M_{\mathrm{Jup}}$ object with an age of either 10 or $50 \mathrm{Myr}$ based on theoretical predictions from the COND models (Baraffe et al. 2003) or

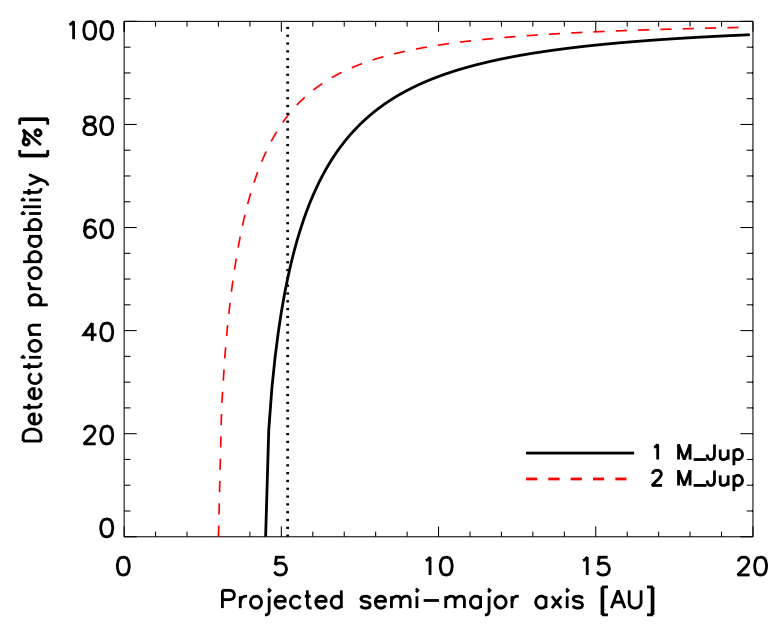

Figure 4. Detection probability of a $1 M_{\text {Jup }}$ object (black, solid line) and a $2 M_{\text {Jup }}$ object (red, dashed line) for our single epoch VLT/NACO observation assuming the detection limits from Figure 2 (left). The vertical, dashed line indicates the position of Jupiter in our solar system at 5.2 AU.

(A color version of this figure is available in the online journal.)

more recent models published by Spiegel \& Burrows (2012). The COND models predict that even for an age of 50 Myr we would have been able to detect objects with masses between 0.5 and $1 M_{\text {Jup }}$ for separations $\geqslant 5$ AU. The models by Spiegel \& Burrows (2012), however, predict that planets are significantly fainter in $L^{\prime}$ compared to the COND models, regardless of their formation history (hot versus cold start models). In this case, only for young ages $(\lesssim 20-30 \mathrm{Myr})$ are our detection limits sufficient to detect a $1 M_{\text {Jup }}$ object in the background limit. For an assumed age of $40 \mathrm{Myr}$, only objects with masses $\geqslant 2 M_{\mathrm{Jup}}$ would have been detected.

\section{DISCUSSION}

Our detection limits are, to our knowledge, the deepest yet achieved for a star targeted by direct imaging in terms of companion mass at a given physical separation. Our results also illustrate an important challenge in interpreting (detections and) non-detections from high-contrast surveys: the systematic and strong dependence upon thermal evolutionary model predictions (see right panel in Figure 2). The Spiegel \& Burrows (2012) models consistently predict fainter $L$-band magnitudes for an $1 M_{\text {Jup }}$ object compared to the Baraffe et al. (2003) COND models, most likely as a result of different assumptions regarding atmospheric opacities (D. S. Spiegel 2012, private communication). As mentioned in the Introduction, presently available atmospheric models fail to self-consistently explain the colors and luminosity of young, low surface gravity objects (e.g., Mohanty et al. 2007; Skemer et al. 2011, 2012; Barman et al. 2011).

Nevertheless, our observations demonstrate that AP Col is unlikely to host a gas giant at separations $\geqslant 5$ AU (Figure 4). For comparison, Delorme et al. (2012) have observed 14 nearby young M-stars, each resulting in non-detections. We note, however, that the highest detection probability of that survey was achieved at separations $\gtrsim 30$ AU. Monte Carlo simulations of current and planned high-contrast surveys indeed indicate that low-mass stars are least likely to harbor a directly detectable planet (Crepp \& Johnson 2011). These simulations are based on empirical results from radial velocity studies that find occurrence rates of $\lesssim 2 \%$ for giant planets with close-in 
orbits (Johnson et al. 2007; Cumming et al. 2008; Bonfils et al. 2011).

However, microlensing results suggest that $17_{-9}^{+6} \%$ of lowmass stars host a gas giant planet with mass between 0.3 and $10 M_{\text {Jup }}$ at separations between 0.5 and $10 \mathrm{AU}$ (Cassan et al. 2012). Thus, the planet frequency seems to rise between the orbital separations probed by Doppler observations and those by microlensing. Combining the currently available results from radial velocity, microlensing, and direct imaging, Quanz et al. (2012) have shown that the population of gas giant planets with semimajor axes between $\sim 0.03-30$ AU can be described by $d f_{\text {Planet }}=M^{\alpha} a^{\beta} d M d a$, with $M$ being the planets' mass, $a$ their semimajor axis, and $\alpha=-1.31$ (cf. Cumming et al. 2008) and $\beta \lesssim 0.5-0.6$. To further constrain the value for $\beta$ and the orbital separation range where it is valid, additional direct imaging observations effectively probing the regions between $\sim 10-30$ AU are required.

\section{SUMMARY AND CONCLUSIONS}

Our findings can be summarized as the following.

1. We have used two different telescopes to obtain deep, highcontrast, $L$-band observations of the nearest $(8.4 \mathrm{pc})$ premain-sequence star, AP Col, to search for planetary mass companions. Neither data set revealed the presence of a candidate companion.

2. Our derived detection limits are the most sensitive yet obtained by direct imaging in terms of planet mass for a given physical separation. For an assumed age of $40 \mathrm{Myr}$, we could have identified objects with masses $\gtrsim 2 M_{\text {Jup }}$ at separations between 3 and $4.5 \mathrm{AU}$, and $\gtrsim 0.5-1 M_{\text {Jup }}$ for separations $\geqslant 4.5 \mathrm{AU}$ using the predicted brightness of planets according to the COND models.

3. Accounting for the unknown inclination and position of a potential planet, we had a $\sim 50 \%$ chance to directly image a $1 M_{\text {Jup }}$ object at 5.2 AU, provided a young Jupiter analog exists.

4. Our detection limits depend on the predicted $L$-band magnitudes for young, planetary mass objects. Using two different sets of theoretical model predictions, the limits change significantly, demonstrating that (1) care need be taken for the interpretation of direct imaging results, and (2) empirical model constraints are urgently required.

Dedicated imaging surveys for planetary mass companions around M-stars are ongoing (e.g., Delorme et al. 2012). They will eventually provide stringent constraints on the overall frequency and semimajor axis distribution of gas giant planets at separations $\gtrsim 10 \mathrm{AU}$, complementing the results of radial velocity and microlensing studies. Our results support the notion that future high-contrast imaging programs would maximize their yield by preferentially selecting massive (A-, F-type) stars as targets (Crepp \& Johnson 2011).

We thank ESO for granting us Director's Discretionary Time for our VLT/NACO observations and are indebted to Lowell Tacconi-Garman and Julien Girard for their excellent support during the observing run. S.P.Q. thanks D. Spiegel for useful discussions about theoretical models of planetary atmospheres. This research has made use of the SIMBAD database, operated at CDS, Strasbourg, France, and of NASA's Astrophysics Data System.

Facilities: VLT:Yepun (NACO), Keck:II (NIRC2)

\section{REFERENCES}

Baraffe, I., Chabrier, G., Barman, T. S., Allard, F., \& Hauschildt, P. H. 2003, A\&A, 402, 701

Barman, T. S., Macintosh, B., Konopacky, Q. M., \& Marois, C. 2011, ApJ, 733, 65

Bergfors, C., Brandner, W., Janson, M., et al. 2010, A\&A, 520, A54 Beuzit, J.-L., Feldt, M., Dohlen, K., et al. 2006, Messenger, 125, 29 Biller, B. A., Close, L. M., Masciadri, E., et al. 2007, ApJS, 173, 143 Bonfils, X., Delfosse, X., Udry, S., et al. 2011, arXiv:1111.5019

Bowler, B. P., Liu, M. C., Dupuy, T. J., \& Cushing, M. C. 2010, ApJ, 723, 850

Cassan, A., Kubas, D., Beaulieu, J.-P., et al. 2012, Nature, 481, 167

Chauvin, G., Lagrange, A., Bonavita, M., et al. 2010, A\&A, 509, A52

Chauvin, G., Lagrange, A., Dumas, C., et al. 2005, A\&A, 438, L25

Cox, A. N. (ed.) 2000, Allen's Astrophysical Quantities (New York: SpringerVerlag)

Crepp, J. R., \& Johnson, J. A. 2011, ApJ, 733, 126

Crepp, J. R., Johnson, J. A., Fischer, D. A., et al. 2012, ApJ, 751, 97

Cumming, A., Butler, R. P., Marcy, G. W., et al. 2008, PASP, 120, 531

Cutri, R. M., Skrutskie, M. F., van Dyk, S., et al. (ed.) 2003, 2MASS All Sky Catalog of Point Sources, http://irsa.ipac.caltech.edu/applications/Gator

Delorme, P., Lagrange, A. M., Chauvin, G., et al. 2012, A\&A, 539, 72 Esposito, S., Mesa, D., Skemer, A., et al. 2012, arXiv:1203.2735

Ghez, A. M., Salim, S., Weinberg, N. N., et al. 2008, ApJ, 689, 1044

Gould, A., Dong, S., Gaudi, B. S., et al. 2010, ApJ, 720, 1073

Heinze, A. N., Hinz, P. M., Kenworthy, M., et al. 2010, ApJ, 714, 1570

Hinkley, S., Oppenheimer, B. R., Zimmerman, N., et al. 2011, PASP, 123,74

Hinz, P. M., Rodigas, T. J., Kenworthy, M. A., et al. 2010, ApJ, 716, 417

Janson, M., Bergfors, C., Goto, M., Brandner, W., \& Lafrenière, D. 2010, ApJ, 710, L35

Johnson, J. A., Aller, K. M., Howard, A. W., \& Crepp, J. R. 2010, PASP, 122 , 905

Johnson, J. A., Butler, R. P., Marcy, G. W., et al. 2007, ApJ, 670, 833

Kasper, M., Apai, D., Janson, M., \& Brandner, W. 2007, A\&A, 472, 321

Kennedy, G. M., \& Kenyon, S. J. 2008, ApJ, 673, 502

Lafrenière, D., Doyon, R., Marois, C., et al. 2007a, ApJ, 670, 1367

Lafrenière, D., Marois, C., Doyon, R., Nadeau, D., \& Artigau, É. 2007b, ApJ, 660,770

Lagrange, A.-M., Bonnefoy, M., Chauvin, G., et al. 2010, Science, 329, 57

Lenzen, R., Hartung, M., Brandner, W., et al. 2003, Proc. SPIE, 4841, 944

Macintosh, B., Graham, J., Palmer, D., et al. 2007, Comptes Rendus Phys., 8 , 365

Marois, C., Lafrenière, D., Doyon, R., Macintosh, B., \& Nadeau, D. 2006, ApJ, 641, 556

Marois, C., Macintosh, B., Barman, T., et al. 2008, Science, 322, 1348

Marois, C., Zuckerman, B., Konopacky, Q. M., Macintosh, B., \& Barman, T. 2010, Nature, 468, 1080

Masciadri, E., Mundt, R., Henning, T., Alvarez, C., \& Barrado y Navascués, D. 2005, ApJ, 625, 1004

Mohanty, S., Jayawardhana, R., Huélamo, N., \& Mamajek, E. 2007, ApJ, 657, 1064

Pollack, J. B., Hubickyj, O., Bodenheimer, P., et al. 1996, Icarus, 124, 62

Pueyo, L., Crepp, J. R., Vasisht, G., et al. 2012, ApJS, 199, 6

Quanz, S. P., Kenworthy, M. A., Meyer, M. R., Girard, J. H. V., \& Kasper, M. 2011, ApJ, 736, L32

Quanz, S. P., Lafrenière, D., Meyer, M. R., Reggiani, M. M., \& Buenzli, E. 2012, A\&A, 541, 133

Quanz, S. P., Meyer, M. R., Kenworthy, M. A., et al. 2010, ApJ, 722, L49

Riedel, A. R., Murphy, S. J., Henry, T. J., et al. 2011, AJ, 142, 104

Rousset, G., Lacombe, F., Puget, P., et al. 2003, Proc. SPIE, 4839, 140

Skemer, A. J., Close, L. M., Szúcs, L., et al. 2011, ApJ, 732, 107

Skemer, A. J., Hinz, P. M., Esposito, S., et al. 2012, ApJ, 753, 14

Spiegel, D. S., \& Burrows, A. 2012, ApJ, 745, 174

Tamura, M. 2009, in AIP Conf. Proc. 1158, Exoplanets and Disks: Their Formation and Diversity, ed. T. Usuda, M. Tamura, \& M. Ishii (Melville, NY: AIP), 11

Wizinowich, P. L., Le Mignant, D., Bouchez, A., et al. 2004, Proc. SPIE, 5490,1 